\title{
Clinical data combined with radiological imaging improves the accuracy of TNM staging of pancreatic body and tail adenocarcinoma
}

This article was published in the following Dove Press journal:

Patient Preference and Adherence

4 October 2017

Number of times this article has been viewed

Wei Xu*

Bo Jiang*

Xinmin Yin*

Department of Hepatobiliary Surgery, Hunan Provincial People's Hospital,

Changsha, China

*These authors contributed equally to this work
Correspondence: Bo Jiang

Department of Hepatobiliary Surgery, Hunan Provincial People's Hospital, No 61 West Jiefang Road, Changsha 410005, China

Tel $+86 \quad 13017286395$

Fax +8673182278012

Email jiangbohnsrmyy@163.com
Purpose: Pancreatic body and tail adenocarcinoma (PBTA) remains one of the deadliest cancers, and current radiological modalities still have limitations on the staging of PBTA. Improving PBTA staging will contribute to the management of this disease.

Patients and methods: Clinicopathological characteristics of 91 surgically treated PBTA patients were retrospectively retrieved. Clinical data associated with postoperative tumor staging (pTNM) were assessed using ordinal logistic regression model. Discriminant analysis was performed using function formula based on multivariate analysis results; further cross-validation was conducted by Bootstrap methods.

Results: Multivariate analysis showed that carbohydrate antigen 19-9 $\geq 955.0 \mathrm{U} / \mathrm{L}$, albumin, and alkaline phosphatase/total bilirubin ratio were independent factors contributing to improved accuracy of pTNM staging. Discriminant analysis exhibited better performance and showed that the probability of accurate prediction of pTNM stage was $90.6 \%$ and the probability of cross-validation was $85.9 \%$. After excluding patients with preoperative diagnosis of stage IV disease, the probability of accurate prediction of pTNM stage was $86.1 \%$ and the probability of cross-validation was $75.0 \%$.

Conclusion: The combination of imaging and clinical data has higher accuracy in staging PBTA than radiological data alone. A model proposed in this study will improve the management of PBTA.

Keywords: pancreatic cancer, pancreatic body and tail adenocarcinoma, TNM staging, diagnostic imaging

\section{Introduction}

Pancreatic cancer is a generic designation for cancer arising in the pancreas and includes a number of subtypes with different cell origins. ${ }^{1}$ Pancreatic adenocarcinoma is the most common subtype, and "pancreatic cancer" is commonly used to indicate this subtype. ${ }^{2,3}$ Patients with pancreatic cancer have poor prognosis with 5-year survival rate $<5 \%$. ${ }^{4,5}$ Therefore, it is urgent to develop diagnosis and therapy for pancreatic cancer. ${ }^{6,7}$ Based on the tumor location, pancreatic cancer can be divided into two types, designated as "pancreatic head adenocarcinoma (PHA)" and "pancreatic body and tail adenocarcinoma (PBTA)" ${ }^{8}$ Compared to PHA, PBTA is less frequent and associated with poorer outcomes. ${ }^{8-10}$ Evidence increasingly suggests that PBTA is quite different from PHA not only in biological behavior but also in disease progression and outcomes. ${ }^{11,12}$ Therefore, further studies on PBTA would be necessary for successful management of patients with PBTA. 
Surgical tumor resection remains the best therapy modality for PBTA patients without metastasis. ${ }^{10,13}$ Surgical indication largely depends on accurate staging of the disease ${ }^{14-17}$ Currently, PBTA staging is based on radiological and endoscopic evaluations and they have similar advantages and disadvantages in tumor staging. ${ }^{18}$ Imaging techniques such as computed tomography (CT) and magnetic resonance imaging (MRI) are of limited benefit for the diagnosis of lymph node metastasis or superior mesenteric artery (SMA) and celiac trunk. ${ }^{19}$ Endoscopic ultrasonography (EUS) has limitation in distinguishing lymph node metastasis from inflammatory hyperplasia, and the technique is largely operator dependent. ${ }^{20-22}$ Although laparoscopy enables the identification of obscure and occult metastases, it is invasive with possible anesthesia-associated complications restricting its routine application. ${ }^{23,24}$ Thus, radiological and endoscopic examinations have limitation in accurate TNM staging of PBTA.

Recently, molecular biomarkers have provided help with cancer staging including pancreatic adenocarcinoma, but their reliability is still a major concern. ${ }^{25,26}$ Until now, carbohydrate antigen 19-9 (CA19-9) has been a very common marker for pancreatic cancer staging, but its expression levels vary greatly in different patients. ${ }^{27}$ Furthermore, CA19-9 is expressed in some benign diseases such as thyroiditis, bile duct stones, obstructive jaundice, and acute pancreatitis as well as several malignant cancers associated with the gallbladder and bile duct. ${ }^{28}$ Thus, CA19-9 is not a perfect marker for pancreatic cancer. The development of a simple and reliable model that can be used in clinical staging of PBTA will greatly improve clinical management. In present study, we established several discriminant functions based on preoperative TNM stage, albumin (ALB), alkaline phosphatase (ALP)/total bilirubin (TBIL) ratio, and CA19-9 to predict the TNM stage of PBTA. Our findings indicated that the combination of radiological imaging and clinical data has higher accuracy for the prediction of TNM stage than imaging alone.

\section{Patients and methods}

\section{Patients}

All PBTA patients were recruited from January 2006 to January 2016. Clinicopathological data were retrospectively retrieved from the electronic database. Patients meeting any of the following criteria were excluded: 1) the primary location of tumor in the pancreas was hard to distinguish; 2) tumor mass affecting the whole pancreas; 3) tumor origin was not clear even after surgery, although PBTA was highly suspected; 4) exact tumor stage could not be diagnosed because surgery was contraindicated; 5) patient was surgically unfit, although the tumor resection was indicated radiologically; and 6) unclear histological diagnosis even after surgery, or pathological analysis revealed a diagnosis other than adenocarcinoma. This study was approved by the Medical Ethics Committee of Hunan Provincial People's Hospital. Written informed consent was obtained from all patients or their families.

\section{Preoperative evaluation}

Only the most recent preoperative results and imaging analyses before surgery were considered in this study. Preoperative evaluation included physical examination, routine blood biochemistry, detection of tumor markers such as carcinoembryonic antigen (CEA) and CA19-9, chest radiography, abdominal ultrasound, $\mathrm{CT}$, MRI, or magnetic resonance cholangiopancreatography. In the majority of cases, preoperative stage was determined by 64-row multidetector CT. A 3.0 T MRI was used for preoperative staging in patients intolerant to intravenous contrast medium for CT. Preoperatively "undefined PBTA" cases mentioned in this study were defined as follows: 1) there was an absence of imaging-detectable tumor mass in the pancreatic body or tail; 2) pancreatic pseudocyst formation after acute pancreatitis was radiologically diagnosed with no evidence of PBTA; 3) imaging showed a space-occupying lesion in the left upper abdomen that could not be localized in the pancreatic body or tail; or 4) PBTA was identified during surgery for other abdominal disorders. The standardized reconstructed unenhanced $5 \mathrm{~mm}$ axial images, pancreatic phase $2.5 \mathrm{~mm}$ axial images, and portal venous phase $5 \mathrm{~mm}$ axial images were generated and analyzed by a resident radiologist and an attending radiologist in a blind manner.

\section{Surgical procedure}

All surgical procedures were conducted following informed consent of the patients and their families. In potentially resectable cases, operative intervention was made to exclude metastatic disease prior to resection. Intraoperative findings were considered the most reliable factors for determining further management. The abdominal cavity was examined such as by intraoperative ultrasonography of the liver to detect radiographically occult peritoneal or liver metastasis. Neoplasms with direct invasion of adjacent major arteries (such as the celiac artery and SMA) confirmed by frozen section examination were considered stage III tumors. Multivisceral resections and suitable venous reconstruction 
were performed whenever necessary to resect all viable cancer cells.

Left-sided pancreatectomy/distal pancreatectomy with splenectomy was performed in potentially resectable patients, while in some patients, en bloc resection (including locally involved structures) and/or venous reconstruction were performed to achieve R0 resection. Peripancreatic lymph nodes were dissected and collected for pathological examination to accurately restage patients. Patients with micrometastases of the liver and peritoneum underwent palliative surgery instead of the scheduled resection. Palliative operations included biliary drainage, gastrojejunostomy, gastrojejunostomy combined with hepatojejunal bypass, T-tube biliary drainage, and laparotomy. The resection margin status was pathologically examined and classified into three groups including R0 (no microscopic residual tumor), R1 (microscopic residual tumor), and R2 (macroscopic residual tumor). Patients with unresectable locoregional (stage III) or metastatic PBTA (stage IV) identified by preoperative imaging were scheduled to receive palliative hepatojejunal and/or gastrojejunal bypass as well as pathological confirmation of PBTA. Appropriate postoperative adjuvant therapy such as chemotherapy, radiotherapy, and radiochemotherapy was suggested in all patients with histologically proven PBTA.

\section{Tumor staging and pathological diagnosis}

The tumor stage was determined based on American Joint Committee on Cancer TNM staging system. ${ }^{29}$ In patients who had resection, pathological diagnosis was based on the resected specimen. In patients who underwent palliative or only exploration procedures, biopsy specimen was taken for pathological diagnosis.

\section{Statistical analysis}

Measurement data are presented as median. For continuous variables, receiver operating characteristic (ROC) curve analysis with area under the curve, sensitivity, and specificity was used to determine the cutoff values. Logistic regression was used to conduct univariate and multivariate analyses of the factors that affect TNM stage. After multivariate analysis, the discriminant functions were established by Bayesian discriminant analysis. The performance of the established discriminant functions was determined by a Bootstrap method, which has been successfully used for estimating the sampling distribution from a limited data set and the statistical properties of the derived quantities. ${ }^{30}$ The accuracy of pTNM staging using the established discriminant functions was compared with that of the simple radiological
TNM staging. Statistical analysis was performed with PASW software Version 18.0, and $P<0.05$ was considered significant difference.

\section{Results \\ Patient characteristics}

This retrospective study was performed from January 2006 to January 2016 at our hospital. During that period, a total of 197 PBTA patients were admitted into our department. Ninety-one patients were eventually recruited into this study and others were excluded due to the following reasons: 1) imaging and surgery could not determine the primary tumor site in the pancreas (11 cases); 2) tumor involved the whole pancreas (three cases); 3) the origin of tumor could not be determined even after surgery (six cases); 4) patients' comorbidities did not allow or patients declined surgery (14 cases); 5) preoperative imaging staged the tumor at TNM IV in the absence of definitive pathological evidence (69 cases); and 6) failure to achieve histological diagnosis even after surgery or pathological diagnosis other than adenocarcinoma (three cases). Data of 91 enrolled patients are shown in Table 1 . The male/female ratio was $2.3 / 1$. The average age was $61.8 \pm 9.7$ years (range $37-84$ years), and the mean duration of disease was 3.4 43.1 months (range 0.1-26.0 months). Main symptoms were epigastric pain $(82.4 \%, 75 / 91)$, unexplained weight loss $(47.3 \%, 43 / 91)$, abdominal discomfort $(35.2 \%, 32 / 91)$, jaundice $(11.0 \%$, $10 / 91)$, abdominal mass $(5.5 \%, 5 / 91)$, and combination with pancreatic cyst at the body or tail $(19.8 \%, 18 / 91)$. Surgical findings were used as the criteria for all patients.

\section{Tumor staging and treatment}

The preoperative tumor stage of all the patients except 13 cases was determined before surgery. The percentage of patients in each stage was 1.1\% (stage IA, 1/91), 4.4\% (stage IB, 4/91), 8.8\% (stage IIA, 8/91), 5.5\% (stage IIB, 5/91), 8.8\% (stage III, 8/91), and 57.1\% (stage IV, 52/91) (Table 2). PBTA was diagnosed surgically in 13 preoperatively undefined cases, among which four cases showed no tumor mass during preoperative imaging, three cases showed a pancreatic cyst after acute pancreatitis, three cases presented a mass in the left upper quadrant, but the exact origin could not be determined, one case was treated for gastric tumor, and two cases were treated for bowel obstruction. Postoperative TNM stage was obtained in all patients. The percentage of patients in each pTNM stage was $0 \%$ (stage IA, 0/91), 0\% (stage IB, 0/9), 5.5\% (stage IIA, 5/91), 1.1\% (stage IIB, 1/91), 14.3\% (stage III, 13/91) and 
Table I Patients' clinical profile: pre- and postoperative stages

\begin{tabular}{|c|c|c|c|c|c|c|c|c|c|}
\hline \multirow[t]{2}{*}{ Factor } & \multirow{2}{*}{$\begin{array}{l}\text { Case } \\
\text { number }\end{array}$} & \multicolumn{5}{|c|}{ Pre-TNM stage } & \multicolumn{3}{|l|}{ pTNM stage } \\
\hline & & $\begin{array}{l}\text { Undefined } \\
(n=13)\end{array}$ & $\begin{array}{l}\text { IA (I) + IB (4) } \\
(n=5)\end{array}$ & $\begin{array}{l}I I A(8)+I I B(5) \\
(n=I 3)\end{array}$ & $\begin{array}{l}\text { III } \\
(n=8)\end{array}$ & $\begin{array}{l}\text { IV } \\
(n=52)\end{array}$ & $\begin{array}{l}\text { IIA (5) + IIB (I) } \\
(n=6)\end{array}$ & $\begin{array}{l}\text { III } \\
(n=13)\end{array}$ & $\begin{array}{l}\text { IV } \\
(n=72)\end{array}$ \\
\hline \multirow[t]{2}{*}{ Gender, M/F } & $63 / 28$ & $10 / 3$ & $1 / 0$ & $6 / 2$ & $5 / 3$ & $34 / 18$ & $5 / 0$ & $8 / 5$ & $49 / 23$ \\
\hline & & & $3 / 1$ & $4 / 1$ & & & $1 / 0$ & & \\
\hline \multicolumn{10}{|l|}{ Age (years) } \\
\hline$\leq 50$ & 10 & I & $1 / 0 / 0$ & $1 / 4 / 3$ & 1 & 6 & $1 / 0 / 4$ & 2 & 7 \\
\hline $5 I-60$ & 32 & 3 & $0 / 0 / 4$ & $0 / 3 / 2$ & 2 & 20 & $0 / 1 / 0$ & 3 & 28 \\
\hline$\geq 60$ & 49 & 9 & & & 5 & 26 & & 8 & 37 \\
\hline \multirow[t]{2}{*}{ Duration (months) ${ }^{\mathrm{a}}$} & 2.4 & 1.5 & 1.4 & 3.0 & 5.7 & 2.1 & 1.5 & 4.7 & 2.3 \\
\hline & & & 4.2 & 3.5 & & & 0.9 & & \\
\hline \multirow[t]{2}{*}{ Weight loss $>5 \mathrm{~kg}$, yes $/ \mathrm{no}$} & $4 I / 50$ & $4 / 9$ & $0 / 1$ & $4 / 4$ & $7 / 1$ & $23 / 29$ & $1 / 4$ & $5 / 8$ & $35 / 37$ \\
\hline & & & $0 / 4$ & $3 / 2$ & & & $0 / 1$ & & \\
\hline Preoperative blood sugar & $47 / 44$ & $9 / 4$ & $0 / 1$ & $6 / 2$ & $2 / 6$ & $27 / 25$ & $4 / 1$ & $5 / 8$ & $38 / 34$ \\
\hline$>6.1 \mathrm{mmol} / \mathrm{L}$, yes $/ \mathrm{no}$ & & & $1 / 3$ & $2 / 3$ & & & $0 / 1$ & & \\
\hline \multirow[t]{2}{*}{ With diabetes, yes/no } & $4 / 87$ & $0 / 13$ & $0 / 1$ & $0 / 8$ & $\mathrm{I} / 7$ & $3 / 49$ & $0 / 5$ & $0 / 13$ & $4 / 68$ \\
\hline & & & $0 / 4$ & $0 / 5$ & & & $0 / 1$ & & \\
\hline \multirow{2}{*}{$\begin{array}{l}\text { With acute pancreatitis, } \\
\text { yes/no }\end{array}$} & $5 / 86$ & $1 / 12$ & $0 / 1$ & $2 / 6$ & $0 / 8$ & $|/ 5|$ & $0 / 5$ & $1 / 12$ & $4 / 68$ \\
\hline & & & $0 / 4$ & $1 / 4$ & & & $0 / 1$ & & \\
\hline \multirow{2}{*}{$\begin{array}{l}\text { Main pancreatic duct dilation } \\
>0.3 \mathrm{~cm} \text {, yes } / \text { no }\end{array}$} & $1 \mathrm{I} / 80$ & $2 / 11$ & $0 / 1$ & $0 / 8$ & $0 / 8$ & $8 / 44$ & $0 / 5$ & $0 / 13$ & $11 / 61$ \\
\hline & & & $0 / 4$ & $\mathrm{I} / 4$ & & & $0 / 1$ & & \\
\hline \multirow{2}{*}{$\begin{array}{l}\text { With chronic pancreatitis, } \\
\text { yes/no }\end{array}$} & $27 / 64$ & $6 / 7$ & $0 / 1$ & $3 / 5$ & $3 / 5$ & $14 / 38$ & $1 / 4$ & $6 / 7$ & $20 / 52$ \\
\hline & & & $0 / 4$ & $1 / 4$ & & & $0 / 1$ & & \\
\hline \multirow{2}{*}{$\begin{array}{l}\text { With pancreatic duct stones, } \\
\text { yes/no }\end{array}$} & $1 / 90$ & $1 / 12$ & $0 / 1$ & $0 / 8$ & $0 / 8$ & $0 / 52$ & $0 / 5$ & $0 / 13$ & $|/ 7|$ \\
\hline & & & $0 / 4$ & $0 / 5$ & & & $0 / 1$ & & \\
\hline \multirow[t]{2}{*}{ Preoperative ascites, yes/no } & $23 / 68$ & $1 / 12$ & $0 / 1$ & $1 / 7$ & $\mathrm{I} / 7$ & $17 / 35$ & $0 / 5$ & $1 / 12$ & $22 / 50$ \\
\hline & & & $1 / 3$ & $2 / 3$ & & & $0 / 1$ & & \\
\hline \multirow[t]{2}{*}{ Tumor size $(\mathrm{cm})^{\mathrm{a}, \mathrm{b}}$} & 5.1 & 5.3 & 2.0 & 4.8 & 4.2 & 5.2 & 3.1 & 4.1 & 5.5 \\
\hline & & & 3.5 & 8.3 & & & 10.2 & & \\
\hline \multirow{2}{*}{$\mathrm{ALT}(\mathrm{U} / \mathrm{L})$} & 14.10 & 13.40 & 11.40 & 9.95 & 16.40 & 16.65 & 11.10 & 10.00 & 17.50 \\
\hline & & & 12.50 & 20.20 & & & 13.70 & & \\
\hline AST (U/L) & 30.5 & 30.5 & 14.0 & 21.9 & 24.5 & 34.1 & 25.5 & 22.9 & 31.3 \\
\hline & & & 33.0 & 26.0 & & & 19.0 & & \\
\hline TBIL $\geq 34.0 \mu \mathrm{mol} / \mathrm{L}$, yes $/ \mathrm{no}$ & $10 / 81$ & $1 / 12$ & $0 / 1$ & $1 / 7$ & $0 / 8$ & $6 / 46$ & $2 / 3$ & $0 / 13$ & $8 / 64$ \\
\hline & & & $2 / 2$ & $0 / 5$ & & & $0 / 1$ & & \\
\hline Serum amylase (U/L) & 51.2 & 55.3 & 94.3 & 41.5 & 46.1 & 49.3 & 104.2 & 41.5 & 54.2 \\
\hline & & & 71.7 & 93.8 & & & 68.7 & & \\
\hline $\operatorname{ALB}(g / L)$ & & & & & & & & & \\
\hline$\leq 30$ & 5 & 0 & $0 / 0 / 1$ & $0 / 1 / 7$ & 0 & 4 & $0 / 1 / 4$ & 0 & 5 \\
\hline $30-35$ & 21 & 2 & $0 / 0 / 4$ & $1 / 2 / 2$ & 1 & 15 & $0 / 0 / 1$ & 0 & 20 \\
\hline$\geq 35$ & 65 & II & & & 7 & 33 & & 13 & 47 \\
\hline WBC $\left(\times 10^{9} / L\right)^{a}$ & 7.0 & 6.6 & 4.6 & 6.2 & 5.3 & 8.1 & 4.9 & 6.3 & 7.2 \\
\hline & & & 6.1 & 7.1 & & & 10.3 & & \\
\hline Neutrophils (\%) ${ }^{\mathrm{a}}$ & 70.6 & 69.6 & 66.5 & 71.2 & 60.7 & 72.1 & 68.5 & 66.5 & 70.8 \\
\hline & & & 70.7 & 68.7 & & & 82.3 & & \\
\hline Lymphocytes (\%) & 19.7 & 22.8 & 28.4 & 19.6 & 25.7 & 18.3 & 22.8 & 23.7 & 19.4 \\
\hline & & & 22.7 & 22.3 & & & 10.9 & & \\
\hline $\mathrm{NLR}^{\mathrm{a}}$ & 3.6 & 2.9 & 2.4 & 3.7 & 2.3 & 3.9 & 2.9 & 2.9 & 3.6 \\
\hline & & & 3.2 & 3.1 & & & 7.5 & & \\
\hline PLT $\left(\times 10^{9} / \mathrm{L}\right)$ & 143.0 & 131.0 & 91.0 & 113.5 & 126.0 & 151.0 & 119.0 & 116.0 & 144.0 \\
\hline & & & 155.0 & 140.0 & & & 324.0 & & \\
\hline $\operatorname{ALP}(\mathrm{U} / \mathrm{L})$ & 98.5 & 88.9 & 47.7 & 85.6 & 97.7 & 125.2 & 66.0 & 82.9 & 108.4 \\
\hline & & & 90.5 & 95.0 & & & 109.7 & & \\
\hline ALP/TBIL cutoff & & & & & & & & & \\
\hline$\leq 6.8$ & 28 & 4 & $0 / 0 / 1$ & $4 / 0 / 4$ & 3 & 13 & $1 / 1 / 3$ & 10 & 17 \\
\hline $6.8-7.1$ & 3 & I & $3 / 0 / 1$ & $1 / 0 / 4$ & 1 & 1 & $0 / 0 / 1$ & 0 & 2 \\
\hline$\geq 7.1$ & 60 & 8 & & & 4 & 38 & & 3 & 53 \\
\hline
\end{tabular}


Table I (Continued)

\begin{tabular}{|c|c|c|c|c|c|c|c|c|c|}
\hline \multirow[t]{2}{*}{ Factor } & \multirow{2}{*}{$\begin{array}{l}\text { Case } \\
\text { number }\end{array}$} & \multicolumn{5}{|c|}{ Pre-TNM stage } & \multicolumn{3}{|l|}{ pTNM stage } \\
\hline & & $\begin{array}{l}\text { Undefined } \\
(n=13)\end{array}$ & $\begin{array}{l}\text { IA (I) + IB (4) } \\
(n=5)\end{array}$ & $\begin{array}{l}I I A(8)+I I B(5) \\
(n=13)\end{array}$ & $\begin{array}{l}\text { III } \\
(n=8)\end{array}$ & $\begin{array}{l}\text { IV } \\
(n=52)\end{array}$ & $\begin{array}{l}\text { IIA (5) + IIB (I) } \\
(n=6)\end{array}$ & $\begin{array}{l}\text { III } \\
(n=13)\end{array}$ & $\begin{array}{l}\text { IV } \\
(n=72)\end{array}$ \\
\hline \multirow[t]{2}{*}{ CEA $>5.0 \mu \mathrm{g} / \mathrm{L}$, yes/no } & $4 I / 50$ & $3 / 10$ & $0 / 1$ & $\mathrm{I} / 7$ & $3 / 5$ & $28 / 24$ & $\mathrm{I} / 4$ & $4 / 9$ & $35 / 37$ \\
\hline & & & $3 / 1$ & $3 / 2$ & & & $\mathrm{I} / 0$ & & \\
\hline \multicolumn{10}{|l|}{ CAI9-9 (U/L) } \\
\hline$\leq 37.0$ & 19 & 5 & $0 / 1 / 0$ & $2 / 3 / 3$ & I & 10 & $4 / 1 / 0$ & 5 & 10 \\
\hline $37.0-955.0$ & 26 & 6 & $1 / 3 / 0$ & $0 / 1 / 4$ & I & 11 & $0 / 0 / 1$ & 7 & 18 \\
\hline$\geq 955.0$ & 46 & 2 & & & 6 & 31 & & I & 44 \\
\hline
\end{tabular}

Notes: aMedian. ${ }^{\mathrm{b}}$ Tumor size was defined as the maximum tumor diameter.

Abbreviations: ALB, albumin; ALP, alkaline phosphatase; ALT, glutamic pyruvic transaminase; AST, aspartate aminotransferase; CAI9-9, carcinoembryonic antigen 19-9; CEA, carcinoembryonic antigen; F, female; M, male; NLR, neutrophils/lymphocyte ratio; PA, prealbumin; PLT, platelet; pTNM, postoperative tumor staging; TBIL, total bilirubin; WBC, white blood cell.

$79.1 \%$ (stage IV, 72/91) (Table 2). Radical resection was planned in 39 patients. Eventually, $11(28.2 \%, 11 / 39)$ patients underwent left-sided pancreatectomy combined with splenectomy, among whom two patients also underwent partial gastrectomy and three patients additionally underwent left colon resection. The number of patients in different groups by resection margin status was R0 (27.3\%, 3/11), R1 (9.1\%, $1 / 11)$, and R2 (63.6\%, 7/11). Gastrojejunostomy and biliary drainage were performed in one $(2.6 \%, 1 / 39)$ patient, gastrojejunostomy was performed in three $(7.7 \%, 3 / 39)$ patients, bile duct exploration and T-tube drainage were performed in one $(2.6 \%, 1 / 39)$ patient, and simple laparotomy was performed in $23(59.0 \%, 23 / 39)$ patients.

Preoperative imaging showed TNM stage IV disease in 52 patients. Thus, palliative bypass surgery was planned in these patients, including gastrojejunostomy in 22 cases, jejunostomy in four cases, and colostomy in three cases. The planned bypass surgery was abandoned in 15 cases due to extensive peritoneal metastasis. After further discussion of intraoperative findings with eight patients' family members during the operation, any further surgical intervention was ruled out.

All patients eventually had a histologically proven diagnosis of adenocarcinoma. Final pathology revealed well-differentiated adenocarcinoma in 43 (47.3\%, 43/91) cases, moderately differentiated adenocarcinoma in 14 $(15.4 \%, 14 / 91)$ cases, and poorly differentiated adenocarcinoma in $34(37.4 \%, 34 / 91)$ cases.

\section{Identification of factors affecting PTNM stage}

By ROC curve analysis, we found that ALP was associated with pTNM stages II, III, and IV, the ratio of ALP/TBIL and ALB was associated with pTNM stages III and IV, and CA19-9 was associated with pTNM stages III and IV. WBC, neutrophil percentage, and neutrophil/lymphocyte ratio (NLR) were not associated with pTNM stage (Table 2).

Univariate analysis showed that ALB, PA, ALP, ALP/ TBIL, and CA19-9 $\geq 955.0 \mathrm{U} / \mathrm{L}$ were associated with pTNM stage (Table 2). Multivariate analysis showed that CA19-9 $\geq 955.0$ U/L, ALB level, and ALP/TBIL ratio were independent factors affecting pTNM stage (Table 3).

Based on only radiological data, the probability of accurate prediction of preoperative and postoperative TNM stage was $76.9 \%$ and the probability of cross-validation was $75.8 \%$ (Table 4). After excluding patients with a definite preoperative diagnosis of stage IV disease, the probability of accurate prediction of preoperative and postoperative TNM stage was $41.0 \%$ and the probability of cross-validation was $38.5 \%$ (Table 5).

\section{Evaluation of discriminant functions for each PTNM stage}

Discriminant functions were developed based on multivariate analysis results. Categorical data were assigned the same numeric values as those in multivariate analysis: 1) preoperative TNM stage: undefined, 0; IA, 1; IB, 2; IIA, 3; IIB, 4; III, 5; IV, 6; and 2) CA19-9 $\geq 955.0 \mathrm{U} / \mathrm{L}$ : yes, 1; no, 0. The function for each pTNM stage was as follows: pTNM IIA, $Y=-44.424+1.726 \times$ (preoperative TNM stage) $+2.204 \times$

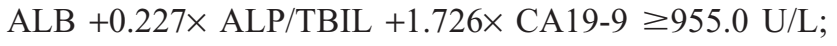
pTNM IIB, $Y=-48.557+1.497 \times$ (preoperative TNM stage $+2.244 \times \mathrm{ALB}+0.296 \times \mathrm{ALP} / \mathrm{TBIL}+6.988 \times$ CA19-9 $\geq 955.0 \mathrm{U} / \mathrm{L}$; pTNM III, $Y=-59.454+2.863 \times$ (preoperative TNM stage) $+2.545 \times$ ALB $+0.244 \times$ ALP/ TBIL +2.044× CA19-9 $\geq 955.0 \mathrm{U} / \mathrm{L}$; and pTNM IV, $Y=-63.497+3.784 \times($ preoperative TNM stage) $+2.512 \times$ ALB +0.305× ALP/TBIL +3.917× CA19-9 $\geq 955.0 \mathrm{U} / \mathrm{L}$. Using these discriminant functions, we calculated the score for each patient based on the preoperative TNM stage, 
Table 2 Univariate analysis of factors associated with pTNM stage

\begin{tabular}{|c|c|c|c|c|c|c|c|}
\hline \multirow[t]{2}{*}{ Factor } & \multirow[t]{2}{*}{ Estimate } & \multirow[t]{2}{*}{ SE } & \multirow[t]{2}{*}{ Wald } & \multirow[t]{2}{*}{$d f$} & \multirow[t]{2}{*}{ Sig } & \multicolumn{2}{|c|}{$95 \%$ confidence interval } \\
\hline & & & & & & Lower bound & Upper bound \\
\hline \multicolumn{8}{|l|}{ Gender } \\
\hline Female & 0.369 & 0.584 & 0.399 & I & 0.528 & -0.776 & 1.513 \\
\hline Male & 0 & & & 0 & & & \\
\hline \multicolumn{8}{|l|}{ Age (years) } \\
\hline$\leq 50$ & -0.275 & 0.755 & 0.133 & I & 0.716 & -1.754 & 1.204 \\
\hline $5 I-60$ & 0.844 & 0.629 & 1.797 & I & 0.180 & -0.390 & 2.077 \\
\hline$\geq 61$ & 0 & & & 0 & & & \\
\hline Disease course (months) & -0.036 & 0.069 & $0.28 I$ & I & 0.596 & -0.171 & 0.098 \\
\hline \multicolumn{8}{|l|}{ Weight loss $>5 \mathrm{~kg}$} \\
\hline No & -0.785 & 0.547 & 2.059 & I & 0.151 & -1.856 & 0.287 \\
\hline Yes & 0 & & & 0 & & & \\
\hline \multicolumn{8}{|l|}{$\mathrm{TBIL} \geq 34.0 \mu \mathrm{mol} / \mathrm{L}$} \\
\hline No & -0.145 & 0.849 & 0.029 & I & 0.864 & -1.810 & 1.519 \\
\hline Yes & 0 & & & 0 & & & \\
\hline \multicolumn{8}{|c|}{ Preoperative blood sugar $>6.1 \mathrm{mmol} / \mathrm{L}$} \\
\hline No & 0.120 & 0.540 & 0.049 & I & 0.825 & -0.940 & 1.179 \\
\hline Yes & 0 & & & 0 & & & \\
\hline \multicolumn{8}{|l|}{ With acute pancreatitis } \\
\hline No & 0.734 & 0.714 & 1.057 & I & 0.304 & -0.666 & 2.134 \\
\hline Yes & 0 & & & 0 & & & \\
\hline \multicolumn{8}{|l|}{ With chronic pancreatitis } \\
\hline No & 0.333 & 0.543 & 0.376 & I & 0.540 & -0.732 & 1.398 \\
\hline Yes & 0 & & & 0 & & & \\
\hline \multicolumn{8}{|l|}{ Preoperative ascites } \\
\hline No & -2.087 & 1.064 & 3.848 & I & 0.050 & -4.171 & -0.002 \\
\hline Yes & 0 & & & 0 & & & \\
\hline \multicolumn{8}{|l|}{ Tumor size $(\mathrm{cm})^{\mathrm{a}}$} \\
\hline Indefinite & -0.818 & 0.588 & 1.933 & I & 0.164 & $-1.97 \mid$ & 0.335 \\
\hline$\leq 2$ & -0.452 & 1.233 & 0.135 & I & 0.714 & -2.869 & 1.964 \\
\hline $2-4$ & -0.679 & 0.763 & 0.791 & I & 0.374 & -2.175 & 0.818 \\
\hline$\geq 4$ & 0 & & & 0 & & & \\
\hline $\mathrm{ALT}(\mathrm{U} / \mathrm{L})$ & 0.016 & 0.012 & 1.747 & I & 0.186 & -0.008 & 0.039 \\
\hline AST (U/L) & 0.030 & 0.017 & 2.966 & I & 0.085 & -0.004 & 0.063 \\
\hline $\operatorname{ALB}(g / L)$ & -0.141 & 0.064 & 4.795 & I & $0.029 *$ & -0.267 & -0.015 \\
\hline Serum amylase (IU/L) & 0.001 & 0.008 & 0.032 & I & 0.858 & -0.014 & 0.016 \\
\hline WBC $\left(\times 10^{9} / L\right)$ & 0.120 & 0.105 & 1.318 & I & 0.251 & -0.085 & 0.325 \\
\hline $\mathrm{HGB}(\mathrm{g} / \mathrm{L})$ & 0.000 & 0.013 & 0.001 & I & 0.975 & -0.026 & 0.027 \\
\hline Neutrophils (\%) & 0.012 & 0.023 & 0.245 & I & 0.621 & -0.034 & 0.058 \\
\hline Lymphocytes (\%) & -0.035 & 0.028 & 1.578 & I & 0.209 & -0.090 & 0.020 \\
\hline NLR & 0.102 & 0.095 & 1.151 & I & 0.283 & -0.084 & 0.288 \\
\hline PLT & 0.001 & 0.004 & 0.055 & I & 0.814 & -0.007 & 0.009 \\
\hline PA & -0.018 & 0.008 & 5.012 & I & $0.025^{*}$ & -0.034 & -0.002 \\
\hline ALP (U/L) & 0.012 & 0.006 & 4.319 & I & $0.038 *$ & 0.001 & 0.024 \\
\hline \multicolumn{8}{|l|}{ ALP/TBIL ratio } \\
\hline$\leq 6.8$ & -1.404 & 0.554 & 6.412 & I & $0.011^{*}$ & -2.490 & -0.317 \\
\hline $6.8-7.1$ & -1.714 & 1.192 & 2.069 & I & 0.150 & -4.050 & 0.622 \\
\hline$\geq 7.1$ & 0 & & & 0 & & & \\
\hline ALP/TBIL ratio & 0.123 & 0.054 & 5.219 & I & $0.022 *$ & 0.018 & 0.230 \\
\hline CEA $>5.0 \mu \mathrm{g} / \mathrm{L}$ & & & & & & & \\
\hline No & -0.668 & 0.555 & 1.446 & I & 0.229 & -1.756 & 0.421 \\
\hline Yes & 0 & & & 0 & & & \\
\hline CAI9-9 (U/mL) & 0.001 & 0.001 & 3.007 & I & 0.083 & -0.000 & 0.002 \\
\hline CAI9-9 $\geq 955.0 \mathrm{U} / \mathrm{mL}$ & & & & & & & \\
\hline No & -2.316 & 0.788 & 8.633 & I & $0.003 *$ & -3.861 & $-0.77 \mid$ \\
\hline Yes & 0 & & & 0 & & & \\
\hline
\end{tabular}

Notes: $* P<0.05$. ${ }^{\mathrm{a}}$ Tumor size was defined as the maximum tumor diameter.

Abbreviations: ALB, albumin; ALP, alkaline phosphatase; ALT, glutamic pyruvic transaminase; AST, aspartate aminotransferase; CAI9-9, carcinoembryonic antigen 19-9; CEA, carcinoembryonic antigen; HGB, hemoglobin; NLR, neutrophils/lymphocyte ratio; PA, prealbumin; PLT, platelet; pTNM, postoperative tumor staging; TBIL, total bilirubin; WBC, white blood cell. 
Table 3 Multivariate analysis of factors associated with pTNM stage

\begin{tabular}{|c|c|c|c|c|c|c|c|}
\hline \multirow[t]{2}{*}{ Factor } & \multirow[t]{2}{*}{ Estimate } & \multirow[t]{2}{*}{ SE } & \multirow[t]{2}{*}{ Wald } & \multirow[t]{2}{*}{$d f$} & \multirow[t]{2}{*}{ Sig } & \multicolumn{2}{|c|}{$95 \%$ confidence interval } \\
\hline & & & & & & Lower bound & Upper bound \\
\hline ALB $(g / L)$ & -0.140 & 0.068 & 4.209 & I & $0.042 *$ & -0.274 & -0.006 \\
\hline ALP & 0.035 & 0.024 & 2.127 & I & 0.145 & -0.012 & 0.081 \\
\hline ALP/TBIL ratio & 0.224 & 0.109 & 4.218 & I & $0.039 *$ & 0.010 & 0.438 \\
\hline \multicolumn{8}{|c|}{ ALP/TBIL ratio cutoff } \\
\hline$\leq 6.8$ & -0.647 & 0.849 & $0.58 \mathrm{I}$ & I & 0.446 & -2.312 & 1.018 \\
\hline $6.8-7.1$ & -1.388 & 1.431 & 0.940 & 1 & 0.332 & -4.192 & 1.417 \\
\hline$\geq 7.1$ & 0 & & & 0 & & & \\
\hline \multicolumn{8}{|c|}{ CAI9-9 $\geq 955.0(\mathrm{U} / \mathrm{L})$} \\
\hline No & -2.258 & 0.875 & 6.664 & I & $0.010 *$ & -3.972 & -0.544 \\
\hline Yes & 0 & & & 0 & & & \\
\hline
\end{tabular}

Note: $* p<0.05$.

Abbreviations: ALB, albumin; ALP, alkaline phosphatase; CA19-9, carcinoembryonic antigen 19-9; pTNM, postoperative tumor staging; TBIL, total bilirubin.

ALP/TBIL, and CA19-9 $\geq 955.0 \mathrm{U} / \mathrm{L}$. The highest score indicated the patient's pTNM stage.

To evaluate the established discriminant functions, we analyzed the patients' TNM stage. Based on multivariate results, we performed Bayesian discriminant analysis and established the discriminant function for each pTNM stage (Table 6). Based on our results, the probability of accurate prediction of pTNM stage was $90.6 \%$ and the probability of cross-validation was $85.9 \%$. After excluding patients with an established preoperative diagnosis of stage IV disease, the probability of accurate prediction of pTNM stage was $86.1 \%$ and the probability of cross-validation was $75.0 \%$ (Table 7). If we simultaneously excluded patients with

Table 4 Cross-validation of pre- and postoperative TNM stages classified by radiological TNM staging system

\begin{tabular}{|c|c|c|c|c|c|}
\hline \multirow{2}{*}{$\begin{array}{l}\text { pTNM } \\
\text { stage }\end{array}$} & \multicolumn{4}{|c|}{ Predicted group membership } & \multirow[t]{2}{*}{ Total } \\
\hline & IIA & IIB & III & IV & \\
\hline \multicolumn{6}{|c|}{ Original } \\
\hline \multicolumn{6}{|l|}{ Count } \\
\hline IIA & 0 & 4 & 1 & 0 & 5 \\
\hline IIB & 0 & I & 0 & 0 & 1 \\
\hline III & 1 & 2 & 7 & 3 & 13 \\
\hline IV & 0 & 6 & 4 & 62 & 72 \\
\hline IIA & 0 & 80 & 20 & 0 & 100 \\
\hline IIB & 0 & 100 & 0 & 0 & 100 \\
\hline III & 7.7 & 15.4 & 53.8 & 23.1 & 100 \\
\hline IV & 0 & 8.3 & 5.6 & 86.1 & 100 \\
\hline \multicolumn{6}{|c|}{ Cross-validated } \\
\hline \multicolumn{6}{|l|}{ Count } \\
\hline$\| \mathrm{A}$ & 0 & 4 & I & 0 & 5 \\
\hline IIB & I & 0 & 0 & 0 & I \\
\hline III & 1 & 2 & 7 & 3 & 13 \\
\hline IV & 0 & 6 & 4 & 62 & 72 \\
\hline IIA & 0 & 80 & 20 & 0 & 100 \\
\hline IIB & 100 & 0 & 0 & 0 & 100 \\
\hline III & 7.7 & 15.4 & 53.8 & 23.1 & 100 \\
\hline IV & 0 & 8.5 & 5.6 & 85.9 & 100 \\
\hline
\end{tabular}

Abbreviation: pTNM, postoperative tumor staging. confirmed preoperative diagnosis of stage IV disease and those with uncertain diagnosis, the probability of accurate prediction of pTNM stage was $92.3 \%$ and the probability of cross-validation was $92.3 \%$ (Table 8). Therefore, the accuracy of TNM staging by our established discriminant functions was much better than that of the procedure based exclusively on radiological data.

\section{Discussion}

In the absence of specific clinical symptoms, most PBTA cases are diagnosed at late stage. ${ }^{31-33}$ Complete surgical resection is an efficient treatment for PBTA patients, and $\mathrm{R} 2$ resection is a negative prognostic factor for postoperative outcome. ${ }^{34-36}$

Table 5 Cross-validation of pre- and postoperative TNM stages classified by radiological TNM staging after excluding patients with a preoperative TNM stage IV disease

\begin{tabular}{|c|c|c|c|c|c|}
\hline \multirow{2}{*}{$\begin{array}{l}\text { pTNM } \\
\text { stage }\end{array}$} & \multicolumn{4}{|c|}{ Predicted group membership } & \multirow[t]{2}{*}{ Tota } \\
\hline & IIA & IIB & III & IV & \\
\hline \multicolumn{6}{|c|}{ Original } \\
\hline \multicolumn{6}{|l|}{ Count } \\
\hline IIA & 0 & 4 & I & 0 & 5 \\
\hline IIB & 0 & I & 0 & 0 & I \\
\hline III & I & 2 & 2 & 8 & 13 \\
\hline IV & 0 & 6 & I & 13 & 20 \\
\hline$\| A$ & 0 & 80 & 20 & 0 & 100 \\
\hline IIB & 0 & 100 & 0 & 0 & 100 \\
\hline III & 7.7 & 15.4 & 15.4 & 61.5 & 100 \\
\hline IV & 0 & 30 & 5 & 65 & 100 \\
\hline \multicolumn{6}{|c|}{ Cross-validated } \\
\hline \multicolumn{6}{|l|}{ Count } \\
\hline IIA & 0 & 4 & I & 0 & 5 \\
\hline IIB & I & 0 & 0 & 0 & I \\
\hline III & I & 2 & 2 & 8 & 13 \\
\hline IV & 0 & 6 & I & 13 & 20 \\
\hline IIA & 0 & 80 & 20 & 0 & 100 \\
\hline IIB & 100 & 0 & 0 & 0 & 100 \\
\hline III & 7.7 & 15.4 & 15.4 & 61.5 & 100 \\
\hline IV & 0 & 30 & 5 & 65 & 100 \\
\hline
\end{tabular}

Abbreviation: PTNM, postoperative tumor staging. 
Table 6 Cross-validation of pre- and postoperative TNM stages classified by Bayesian discriminant functions

\begin{tabular}{llllll}
\hline pTNM & \multicolumn{3}{l}{ Predicted } & & group \\
\cline { 2 - 4 } stage & IIA & IIB & III & IV & \\
\hline
\end{tabular}

\begin{tabular}{llllll}
\hline $\begin{array}{l}\text { Original } \\
\text { Count }\end{array}$ & & & & & \\
IIA & 4 & 0 & 0 & 0 & 4 \\
IIB & 0 & 1 & 0 & 0 & 1 \\
III & 1 & 0 & $I I$ & 0 & 12 \\
IV & 2 & 2 & 3 & $6 I$ & 68 \\
IIA & 100 & 0 & 0 & 0 & 100 \\
IIB & 0 & 100 & 0 & 0 & 100 \\
III & 8.3 & 0 & 91.7 & 0 & 100 \\
IV & 2.9 & 2.9 & 4.4 & 89.8 & 100 \\
Cross-validated & & & & \\
Count & & & & & \\
IIA & 3 & 0 & 1 & 0 & 4 \\
IIB & 1 & 0 & 0 & 0 & 1 \\
III & 1 & 0 & 10 & 1 & 12 \\
IV & 3 & 3 & 2 & 60 & 68 \\
IIA & 75 & 0 & 25 & 0 & 100 \\
IIB & 100 & 0 & 0 & 0 & 100 \\
III & 8.3 & 0 & 83.4 & 8.3 & 100 \\
IV & 4.4 & 4.4 & 2.9 & 88.3 & 100 \\
\hline
\end{tabular}

Abbreviation: PTNM, postoperative tumor staging.

The selection of $\mathrm{R} 2$ resection or complete surgical resection is largely dependent on accurate preoperative diagnosis of disease stage. Currently, PBTA staging is dependent on imaging modalities including CT, MRI, and EUS, particularly

Table 7 Cross-validation of pre- and postoperative TNM stages classified by Bayesian discriminant functions after excluding patients with a preoperative TNM stage IV disease

\begin{tabular}{|c|c|c|c|c|c|}
\hline \multirow{2}{*}{$\begin{array}{l}\text { pTNM } \\
\text { stage }\end{array}$} & \multicolumn{4}{|c|}{ Predicted group membership } & \multirow[t]{2}{*}{ Total } \\
\hline & IIA & IIB & III & IV & \\
\hline \multicolumn{6}{|c|}{ Original } \\
\hline \multicolumn{6}{|l|}{ Count } \\
\hline$\| \mathrm{A}$ & 4 & 0 & 0 & 0 & 4 \\
\hline IIB & 0 & 1 & 0 & 0 & I \\
\hline III & I & 0 & 10 & I & 12 \\
\hline IV & I & 1 & I & 16 & 19 \\
\hline IIA & 100 & 0 & 0 & 0 & 100 \\
\hline IIB & 0 & 100 & 0 & 0 & 100 \\
\hline III & 8.3 & 0 & 83.3 & 8.4 & 100 \\
\hline IV & 5.3 & 5.3 & 5.3 & 84.1 & 100 \\
\hline \multicolumn{6}{|c|}{ Cross-validated } \\
\hline \multicolumn{6}{|l|}{ Count } \\
\hline$\| \mathrm{A}$ & 3 & 0 & I & 0 & 4 \\
\hline IIB & 0 & 0 & 0 & I & I \\
\hline III & I & 0 & 10 & I & 12 \\
\hline IV & 2 & 1 & 2 & 14 & 19 \\
\hline$\| \mathrm{A}$ & 75 & 0 & 25 & 0 & 100 \\
\hline IIB & 0 & 0 & 0 & 100 & 100 \\
\hline III & 8.3 & 0 & 83.3 & 8.4 & 100 \\
\hline IV & 10.5 & 5.3 & 10.5 & 73.7 & 100 \\
\hline
\end{tabular}

Abbreviation: PTNM, postoperative tumor staging.
Table 8 Cross-validation of pre- and postoperative TNM stages classified by Bayesian discriminant functions after excluding patients with preoperative diagnosis of TNM stage IV and undefined PBTA

\begin{tabular}{llllll}
\hline PTNM & \multicolumn{3}{l}{ Predicted group membership } & Total \\
\cline { 2 - 5 } stage & IIA & IIB & III & IV & \\
\hline Original & & & & & \\
Count & & & & & \\
IIA & 2 & 0 & 0 & 2 & 2 \\
III & 0 & 9 & I & 10 & 0 \\
IV & 0 & 1 & 13 & 14 & 0 \\
IIA & I00 & 0 & 0 & 100 & 100 \\
III & 0 & 90 & 10 & 100 & 0 \\
IV & 0 & 7.1 & 92.9 & 100 & 0 \\
Cross-validated & & & & \\
Count & & & & & \\
IIA & 2 & 0 & 0 & 2 & 2 \\
III & 0 & 9 & 1 & 10 & 0 \\
IV & 0 & 1 & 13 & 14 & 0 \\
IIA & I00 & 0 & 0 & 100 & 100 \\
III & 0 & 90 & 10 & 100 & 0 \\
IV & 0 & 7.1 & 92.9 & 100 & 0 \\
\hline A & 0 &
\end{tabular}

Abbreviations: PBTA, pancreatic body and tail adenocarcinoma; PTNM, postoperative tumor staging.

during the initial work-up. ${ }^{37}$ However, current PBTA staging system based on imaging data has several limitations and the development of a simple and reliable method of staging is of great significance in the management of patients with PBTA as well as the improvement of overall outcomes. We hypothesized that the combination of imaging with clinical pathological data provides accurate prediction. Our results showed that preoperative TNM stage, ALB, ALP/TBIL, and CA19-9 $\geq 955.0 \mathrm{U} / \mathrm{L}$ reliably predicted the stage. Based on these factors, we established several functions for each stage of the disease. The probability of accurate prediction of pTNM stage and the probability of cross-validation were $>90 \%$, significantly higher than those obtained using imaging findings alone. Thus, the staging procedure combining imaging and clinical data might be useful for future clinical application.

Despite recently dramatically increased resolution of imaging techniques such as $\mathrm{CT}$ and MRI, $\sim 10 \%$ of primary tumors were not distinguished due to similar density. Furthermore, liver metastasis or peritoneal metastasis was easily misdiagnosed by CT or MRI. ${ }^{38}$ Remarkably, we found 13 patients who had negative findings on abdominal CT examination, although their tumors were refractory to surgical intervention. The presence of chronic pancreatitis has been shown to limit the accuracy of EUS considerably. Furthermore, EUS is limited in its ability to facilitate the 
evaluation of $\mathrm{N}$ stage or the invasion of SMA and celiac artery. ${ }^{21,22}$ Therefore, it is almost impossible to overcome these limitations by using imaging techniques alone. In the present study, despite extensive preoperative evaluation and resections, the exploration rate of $59.0 \%(23 / 39)$ is still high. Identification of peritoneal or liver metastases undetectable with preoperative imaging techniques resulted in aborted surgical resection. In our study, all potentially resectable patients received intention-to-treat curative resection. However, R0 resection rate of only $27.3 \%$ (3/11) and a disappointing higher rate of positive resection margins demonstrated that PBTA has been a diagnostic challenge with imaging modalities. The combined use of CA19-9 and laparoscopy was suggested to avoid unnecessary laparotomies, suggesting that the combination of multiple biologically unrelated factors might be a better approach. ${ }^{23,24}$

CA19-9 was used as a marker for the diagnosis of pancreatic cancer, assessment of response to chemotherapy, evaluation of prognosis, and prediction of tumor recurrence. ${ }^{39}$ However, CA19-9 was associated with significant limitations such as the lack of sensitivity and false-negative or false-positive elevation results. ${ }^{40}$ Therefore, determination of the cutoff level of CA19-9 is important. Here, we found that CA19-9 $\geq 955.0 \mathrm{U} / \mathrm{mL}$ was a sensitive marker to predict the stage of PBTA. For patients with pTNM stage IV disease, the sensitivity and specificity of CA19-9 $\geq 955.0 \mathrm{U} / \mathrm{mL}$ were $58.0 \%$ and $88.2 \%$, respectively. For patients with pTNM stage III disease, the sensitivity and specificity were $55.4 \%$ and $91.7 \%$, respectively. Combining CA19-9 with the other three factors dramatically increased the probability of accurate prediction of PBTA staging. Thus, the combination of multiple factors might overcome the limitations of a single factor.

NLR is an indicator of systemic inflammatory response, and its role as an independent prognostic marker for primary operable PC and advanced PC has been intensively explored. Previous reports showed that NLR was a marker for the prognosis of pancreatic cancer. ${ }^{40}$ In the current study, we tried to analyze the potential application of NLR in the staging of PBTA. Unexpectedly, we failed to find the association between pTNM stages and WBC, neutrophil percentage, or NLR. These findings suggest that pancreatic tumors derived from different locations may have different features. It might also imply a potential biological difference between PHA and PBTA, which needs to be further determined.

Another interesting finding in our study was the identification of two other stage-associated factors, ALP/TBIL ratio and ALB. We found that ALP/TBIL ratio but not ALP or
TBIL alone predicted the PBTA stage, suggesting that the basic levels of ALP and TBIL might be partially regulated by PBTA-independent factors. Therefore, reducing the background variation using ALP/TBIL ratio might facilitate the staging of PBTA. Low ALB is a prognostic marker for pancreatic cancer. ${ }^{41,42}$ Although we found that the four factors could be used to stage PBTA, their exact biological function and functional interaction are still largely unknown. Our findings suggest that the biological behavior and pathological process of PHA and PBTA might be different, despite similar clinical presentation and histological appearance.

As the treatment of PBTA continues to evolve and requires a multimodal approach, accurate staging becomes increasingly important to match therapeutic strategies with disease stage. In our opinion, diagnostic laparoscopy prior to laparotomy and resection is useful in avoiding unnecessary laparotomies in highly selected patients whose preoperative evaluation strongly precludes radical resection and directs them for neoadjuvant chemoradiation. In this regard, our study provided an easy-to-use model, which combines measurable clinical data and radiological findings, to help make clinical decisions.

$\mathrm{CT}$ and MRI and not EUS play a primary role in our PC imaging protocol and tumor staging. The past years witnessed significant improvements in CT technology such as higher image resolution and faster image acquisition. In this study, PBTA diagnosis and staging were based on long-term and heterogeneous CT techniques, which may be a limitation of this study. In addition, several newly developed techniques such as endoscopic ultrasonography (EUS) and positron emission tomography-computed tomography (PET-CT) have been shown to increase the accuracy of PBTA staging. However, these techniques are expensive and not routinely applied in clinical practice. Since only a small portion of our patients underwent preoperative EUS or PET-CT evaluation, we did not include these imaging data in our analysis. Future studies should carefully address these issues.

\section{Conclusion}

By analyzing clinical data of patients with PBTA, we have established the discriminant functions based on preoperative TNM stage, ALB, ALP/TBIL, and CA19-9 to improve the staging accuracy. The current study underscores the importance of radiographic staging combined with clinical data for risk stratification of patients, in tailoring therapy and management. However, our study was based on a limited number of patients and large-scale studies are required to validate our findings. Nonetheless, we believe that the combination of different 
factors to predict the PBTA stage as well as PHA is useful. The combination of multiple, biologically unrelated factors might be useful for staging cancer patients and help overcome the limitations associated with staging based on similar or biologically related factors.

\section{Disclosure}

The authors report no conflicts of interest in this work.

\section{References}

1. Saif MW. Pancreatic neoplasm in 2011: an update. JOP. 2011;12(4): 316-321.

2. Parsons CM, Sutcliffe JL, Bold RJ. Preoperative evaluation of pancreatic adenocarcinoma. J Hepatobiliary Pancreat Surg. 2008;15(4): 429-435.

3. Longo R, Cacciamani F, Naso G, Gasparini G. Pancreatic cancer: from molecular signature to target therapy. Crit Rev Oncol Hematol. 2008; 68(3):197-211.

4. Helmstaedter L, Riemann JF. Pancreatic cancer - EUS and early diagnosis. Langenbecks Arch Surg. 2008;393(6):923-927.

5. Bliss LA, Witkowski ER, Yang CJ, Tseng JF. Outcomes in operative management of pancreatic cancer. J Surg Oncol. 2014;110(5):592-598.

6. Hidalgo M, Cascinu S, Kleeff J, et al. Addressing the challenges of pancreatic cancer: future directions for improving outcomes. Pancreatology. 2015;15(1):8-18.

7. Moniri MR, Dai LJ, Warnock GL. The challenge of pancreatic cancer therapy and novel treatment strategy using engineered mesenchymal stem cells. Cancer Gene Ther. 2014;21(1):12-23.

8. Ling Q, Xu X, Zheng SS, Kalthoff $\mathrm{H}$. The diversity between pancreatic head and body/tail cancers: clinical parameters and in vitro models. Hepatobiliary Pancreat Dis Int. 2013;12(5):480-487.

9. Lau MK, Davila JA, Shaib YH. Incidence and survival of pancreatic head and body and tail cancers: a population-based study in the United States. Pancreas. 2010;39(4):458-462.

10. Brennan MF, Moccia RD, Klimstra D. Management of adenocarcinoma of the body and tail of the pancreas. Ann Surg. 1996;223(5):506-511.

11. Watanabe I, Sasaki S, Konishi M, et al. Onset symptoms and tumor locations as prognostic factors of pancreatic cancer. Pancreas. 2004; 28(2):160-165.

12. Artinyan A, Soriano PA, Prendergast C, Low T, Ellenhorn JD, Kim J. The anatomic location of pancreatic cancer is a prognostic factor for survival. HPB (Oxford). 2008;10(5):371-376.

13. Sahin TT, Fujii T, Kanda M, et al. Prognostic implications of lymph node metastases in carcinoma of the body and tail of the pancreas. Pancreas. 2011;40(7):1029-1033.

14. Pawlik TM, Laheru D, Hruban RH, et al. Evaluating the impact of a single-day multidisciplinary clinic on the management of pancreatic cancer. Ann Surg Oncol. 2008;15(8):2081-2088.

15. Evans DB, Varadhachary GR, Crane CH, et al. Preoperative gemcitabinebased chemoradiation for patients with resectable adenocarcinoma of the pancreatic head. J Clin Oncol. 2008;26(21):3496-3502.

16. Almadi MA, Alharbi O, Azzam N, et al. Clinical predictors of resectability of pancreatic adenocarcinoma. Saudi J Gastroenterol. 2013; 19(6):278-285.

17. Takahashi Y, Kaneoka Y, Maeda A, Isogai M. Distal pancreatectomy with celiac axis resection for carcinoma of the body and tail of the pancreas. World J Surg. 2011;35(11):2535-2542.

18. Bipat S, Phoa SS, van Delden OM, et al. Ultrasonography, computed tomography and magnetic resonance imaging for diagnosis and determining resectability of pancreatic adenocarcinoma: a meta-analysis. J Comput Assist Tomogr. 2005;29(4):438-445.

19. Schima W, Ba-Ssalamah A, Kolblinger C, Kulinna-Cosentini C, Puespoek A, Gotzinger P. Pancreatic adenocarcinoma. Eur Radiol. 2007;17(3):638-649.
20. Iglesias Garcia J, Larino Noia J, Dominguez Munoz JE. Endoscopic ultrasound in the diagnosis and staging of pancreatic cancer. Rev Esp Enferm Dig. 2009;101(9):631-638.

21. Canto MI, Goggins M, Yeo CJ, et al. Screening for pancreatic neoplasia in high-risk individuals: an EUS-based approach. Clin Gastroenterol Hepatol. 2004;2:606-621.

22. Canto MI, Goggins M, Hruban RH, et al. Screening for early pancreatic neoplasia in high-risk individuals: a prospective controlled study. Clin Gastroenterol Hepatol. 2006;4(6):766-781.

23. Contreras CM, Stanelle EJ, Mansour J, et al. Staging laparoscopy enhances the detection of occult metastases in patients with pancreatic adenocarcinoma. J Surg Oncol. 2009;100(8):663-669.

24. Satoi S, Yanagimoto H, Toyokawa H, et al. Selective use of staging laparoscopy based on carbohydrate antigen 19-9 level and tumor size in patients with radiographically defined potentially or borderline resectable pancreatic cancer. Pancreas. 2011;40(3):426-432.

25. Jhaveri DT, Zheng L, Jaffee EM. Specificity delivers: therapeutic role of tumor antigen-specific antibodies in pancreatic cancer. Semin Oncol. 2014;41(5):559-575.

26. Lennon AM, Wolfgang CL, Canto MI, et al. The early detection of pancreatic cancer: what will it take to diagnose and treat curable pancreatic neoplasia? Cancer Res. 2014;74(13):3381-3389.

27. Herreros-Villanueva M, Gironella M, Castells A, Bujanda L. Molecular markers in pancreatic cancer diagnosis. Clin Chim Acta. 2013;418: 22-29.

28. Poruk KE, Gay DZ, Brown K, et al. The clinical utility of CA 19-9 in pancreatic adenocarcinoma: diagnostic and prognostic updates. Curr Mol Med. 2013;13(3):340-351.

29. Helm J, Centeno BA, Coppola D, et al. Histologic characteristics enhance predictive value of American Joint Committee on Cancer staging in resectable pancreas cancer. Cancer. 2009;115(18):4080-4089.

30. Rosenberger WF, Hu F. Bootstrap methods for adaptive designs. Stat Med. 1999;18(14):1757-1767.

31. Fokas E, O’Neill E, Gordon-Weeks A, Mukherjee S, McKenna WG, Muschel RJ. Pancreatic ductal adenocarcinoma: from genetics to biology to radiobiology to oncoimmunology and all the way back to the clinic. Biochim Biophys Acta. 2015;1855(1):61-82.

32. Ryan DP, Hong TS, Bardeesy N. Pancreatic adenocarcinoma. N Engl J Med. 2014;371:1039-1049.

33. Mian OY, Ram AN, Tuli R, Herman JM. Management options in locally advanced pancreatic cancer. Curr Oncol Rep. 2014;16(6):388.

34. Goh BK, Tan YM, Cheow PC, et al. Outcome of distal pancreatectomy for pancreatic adenocarcinoma. Dig Surg. 2008;25(1):32-38.

35. Jazieh KA, Foote MB, Diaz LA Jr. The clinical utility of biomarkers in the management of pancreatic adenocarcinoma. Semin Radiat Oncol. 2014;24(2):67-76.

36. Rucki AA, Zheng L. Pancreatic cancer stroma: understanding biology leads to new therapeutic strategies. World J Gastroenterol. 2014; 20(9):2237-2246.

37. Poruk KE, Firpo MA, Mulvihill SJ. Screening for pancreatic cancer. Adv Surg. 2014;48:115-136.

38. Morgan KA, Adams DB. Solid tumors of the body and tail of the pancreas. Surg Clin North Am. 2010;90(2):287-307.

39. Ballehaninna UK, Chamberlain RS. Biomarkers for pancreatic cancer: promising new markers and options beyond CA 19-9. Tumour Biol. 2013;34(6):3279-3292.

40. Galli C, Basso D, Plebani M. CA 19-9: handle with care. Clin Chem Lab Med. 2013;51(7):1369-1383.

41. Hashimoto K, Ueno H, Ikeda M, et al. Do recurrent and metastatic pancreatic cancer patients have the same outcomes with gemcitabine treatment? Oncology. 2009;77(3-4):217-223.

42. Smith RA, Dajani K, Dodd S, et al. Preoperative resolution of jaundice following biliary stenting predicts more favourable early survival in resected pancreatic ductal adenocarcinoma. Ann Surg Oncol. 2008;15(11):3138-3146. 
Patient Preference and Adherence

Dovepress

\section{Publish your work in this journal}

Patient Preference and Adherence is an international, peer-reviewed, open access journal that focuses on the growing importance of patient preference and adherence throughout the therapeutic continuum. Patient satisfaction, acceptability, quality of life, compliance, persistence and their role in developing new therapeutic modalities and compounds to optimize clinical outcomes for existing disease states are major areas of interest for the journal. This journal has been accepted for indexing on PubMed Central The manuscript management system is completely online and includes a very quick and fair peer-review system, which is all easy to use. Visit http://www. dovepress.com/testimonials.php to read real quotes from published authors.

Submit your manuscript here: http://www.dovepress.com/patient-preference-and-adherence-journal 